\title{
QUASISYMMETRIC FUNCTIONS OF EXTREMAL GROWTH
}

\section{A. HINKKANEN ${ }^{1}$ )}

\section{Introduction}

In this paper we continue the study of the growth of quasisymmetric functions started in [2] and [3] by W. K. Hayman and the author. An increasing homeomorphism $f$ of the real line $R$ onto itself is called $K$-quasisymmetric ( $K$-qs) if

$$
\frac{1}{K} \leqq \frac{f(x+t)-f(x)}{f(x)-f(x-t)} \leqq K
$$

holds for all $x \in R$ and $t>0$. Following [2], we set

$$
N_{0}(K)=\{f \mid f \text { is } K \text {-qs, } f(-1)=-1, f(1)=1\},
$$

where $K \geqq 1$, and

$$
\begin{aligned}
M_{0}(x, K) & =\max \left\{f(x) \mid f \in N_{0}(K)\right\}, \\
m_{0}(x, K) & =\min \left\{f(x) \mid f \in N_{0}(K)\right\} .
\end{aligned}
$$

Note that by [1], the class $N_{0}(K)$ is compact. Qs functions arose as boundary values of quasi-conformal maps [1].

In [2], various estimates for $M_{0}(x, K)$ and $m_{0}(x, K)$ were established. It was shown that there are positive numbers $\alpha_{1}, \alpha_{2}, c_{1}, c_{2}$, depending on $K$ only, such that

$$
\begin{aligned}
& x^{\alpha_{1}} \leqq M_{0}(x, K) \leqq c_{1} x^{\alpha_{1}}, \\
& c_{2} x^{\alpha_{2}} \leqq m_{0}(x, K) \leqq x^{\alpha_{2}}
\end{aligned}
$$

for $x \geqq 1$. Further, Hayman [3] showed that $M_{0}(x, K) x^{-\alpha_{1}}$ and $m_{0}(x, K) x^{-\alpha_{2}}$ tend to a limit as $x \rightarrow \infty$ if $(\log K) /(\log (K+1) / 2)$ is irrational, while if this ratio is rational, then $M_{0}(x, K) x^{-\alpha_{1}}$ and $m_{0}(x, K) x^{-\alpha_{2}}$ are asymptotic to a periodic function $\varphi$ of $\log x$ as $x \rightarrow \infty$. The question whether or not $\varphi$ is constant was left open, as well as the problem of the value of the limit.

In this paper we shall construct a piecewise linear function $f \in N_{0}(K)$, which turns out to be the largest convex minorant of $M_{0}(x, K)$ for $x \geqq-1$. A similar

1) Research supported by the Osk. Huttunen Foundation, Helsinki. 
construction leads to a function $g \in N_{0}(K)$, which is the smallest concave majorant of $m_{0}(x, K)$ for $x \geqq-1$. There are infinitely many points $x$ such that $f(x)=$ $M_{0}(x, K)$ or $g(x)=m_{0}(x, K)$. If $(\log K) /(\log (K+1) / 2)$ is rational, these points $x$ occur at bounded distances.

Since $f$ and $g$ are defined by means of explicit formulas, these results can be used to obtain information about the asymptotic behaviour of $M_{0}(x, K)$ and $m_{0}(x, K)$. For example, one can determine the above function $\varphi$ and show that it is not constant. These applications will be developed in a later paper.

It turns out (see Theorem 3 below and cf. [2, Theorem 13]) that if $K>1$, then there are infinitely many rational values $x_{0}$ such that $M_{0}(x, K)$ is not differentiable at $x=x_{0}$. Since $M_{0}(x, K)$ is increasing, it is differentiable almost everywhere. One could suggest that such a special behaviour at certain rational points might partly be due to the way we have chosen to define qs functions by means of (1.1) (i.e., by considering the line segments $(x-t, x)$ and $(x, x+t)$ of equal length, while to obtain $M_{0}(x, K)$ one essentially considers line segments whose lengths have the ratio $(x-1) / 2)$. However, it is not so clear why there should be a qualitative difference in the asymptotic behaviour of $M_{0}(x, K)$ for different values of $K$. So perhaps the most interesting feature of the results is that they allow us to study these somewhat unexpected irregularities of extremal growth in greater detail, even though it may be remarked that less precise information is usually sufficient for applications.

Next we define the functions $f$ and $g$ and then state the theorems containing our results.

For $K \geqq 1$, we set $L=L(K)=(K+1) / 2$ and $\Lambda=\Lambda(K)=\left(K^{-1}+1\right) / 2$. We number the pairs $(p, q)$ of integers $p \geqq 1, q \geqq 0$ so that

$$
K^{p_{1}} L^{q_{1}} \leqq K^{p_{2}} L^{q_{2}} \leqq \ldots
$$

Such an ordering is not unique if and only if $K^{q}=L^{p}$ for some $p$ and $q$, but in this case any ordering satisfying (1.2) could be chosen. However, to be definite, in the proof of Theorem 1 the particular order, in which the pairs $(p, q)$ for which the numbers $K^{p} L^{q}$ are equal are taken in the descending order of $p$, will be considered.

As usual, we write $\left(\begin{array}{c}m \\ n\end{array}\right)=m ! /(n !(m-n) !)$.

We set $X_{0}=1$ and, for $n \geqq 1$, we set

$$
X_{n}=1+\sum_{k=1}^{n}\left(\begin{array}{c}
p_{k}+q_{k}-1 \\
q_{k}
\end{array}\right) 2^{q_{k}+1} .
$$

We define $f(0)=0, f(1)=1$, and

$$
f\left(X_{n}\right)=1+2 \sum_{k=1}^{n}\left(\begin{array}{c}
p_{k}+q_{k}-1 \\
q_{k}
\end{array}\right) K^{p_{k}}(K+1)^{q_{k}}, \quad n \geqq 1 .
$$


We take $f$ to be continuous and linear on $\left[X_{n-1}, X_{n}\right], n \geqq 1$, and we set $f(x)=x$, $0 \leqq x \leqq 1$, and $f(-x)=-f(x)$. Thus the slope of $f$ on $\left[X_{n-1}, X_{n}\right]$ equals $K^{p_{n}} L^{q_{n}}$.

Similarly, we number the pairs $(P, Q), P \geqq 1, Q \geqq 0$, so that

$$
K^{-P_{1}} \Lambda^{Q_{1}} \geqq K^{-P_{2}} \Lambda^{Q_{2}} \geqq \ldots
$$

We set $Y_{0}=1$,

$$
\begin{gathered}
Y_{n}=1+\sum_{k=1}^{n}\left(\begin{array}{c}
P_{k}+Q_{k}-1 \\
Q_{k}
\end{array}\right) 2^{Q_{k}+1}, \\
g\left(Y_{n}\right)=1+2 \sum_{k=1}^{n}\left(\begin{array}{c}
P_{k}+Q_{k}-1 \\
Q_{k}
\end{array}\right) K^{-P_{k}}(1+1 / K)^{Q_{k}},
\end{gathered}
$$

and take $g$ to be linear and continuous on each $\left[Y_{n-1}, Y_{n}\right]$. Further, we set $g(x)=x$, $0 \leqq x \leqq 1$, and $g(-x)=-g(x)$. Thus the slope of $g$ on $\left[Y_{n-1}, Y_{n}\right]$ equals $K^{-P_{n}} \Lambda^{Q_{n}}$.

Now we are ready to state our results.

Theorem 1. Suppose that $K>1$. Then $f$ and $g$ belong to $N_{0}(K)$. We have

$$
f(x)=M_{0}(x, K)
$$

for $x=X_{n-1}+j 2^{q_{n}+1}$ for all $n \geqq 1$ and $0 \leqq j \leqq\left(\begin{array}{c}p_{n}+q_{n}-1 \\ q_{n}\end{array}\right)$, and

$$
g(x)=m_{0}(x, K)
$$

for $x=Y_{n-1}+j 2^{Q_{n}+1}$ for all $n \geqq 1$ and $0 \leqq j \leqq\left(\begin{array}{c}P_{n}+Q_{n}-1 \\ Q_{n}\end{array}\right)$.

From Theorem 1 we deduce the following results:

Theorem 2. For a fixed $K$, the function $f$ is the largest convex minorant of $M_{0}(x, K)$ and the function $g$ is the smallest concave majorant of $m_{0}(x, K)$ for $x \geqq-1$.

Theorem 3. If the slope of $f$ changes at $x=X_{n}$, then $M_{0}(x, K)$ is not differentiable as a function of $x$ at $x=X_{n}$. If the slope of $g$ changes at $x=Y_{n}$, then $m_{0}(x, K)$ is not differentiable as a function of $x$ at $x=Y_{n}$.

It turns out that if $(\log K) /(\log [(K+1) / 2])$ is rational, then there are more points $x$ than those mentioned in Theorem 1 where (1.8) or (1.9) is valid.

Theorem 4. Suppose that $K>1$ and that

$$
\frac{\log K}{\log [(K+1) / 2]}=\frac{p}{q}
$$

where $p$ and $q$ are positive, relatively prime integers. Then if $z_{1} \geqq 1$ and $f\left(z_{1}\right)=$ $M_{0}\left(z_{1}, K\right)$, there is a point $z_{2}$ with $f\left(z_{2}\right)=M_{0}\left(z_{2}, K\right)$ and

$$
z_{1}<z_{2} \leqq z_{1}+2^{2^{p}+p} .
$$


Further if $z_{3} \geqq 1$ and $g\left(z_{3}\right)=m_{0}\left(z_{3}, K\right)$, there is a point $z_{4}$ with $g\left(z_{4}\right)=m_{0}\left(z_{4}, K\right)$ and

$$
z_{3}<z_{4} \leqq z_{3}+2^{2^{p}+2 p} .
$$

If the left hand side of (1.10) is irrational, Theorem 1 implies that between $X_{n-1}$ and $X_{n}$, there are points $x$, where (1.8) holds, at a distance $2^{q_{n}+1}$ from each other. As $n \rightarrow \infty$, this distance has lower limit 2 and upper limit $\infty$.

Theorems 1 and 3 may be considered as extensions of [2, Theorem 13]. There we had the first few pieces of the present functions $f$ and $g$.

In Sections 2-6 we prove Theorems 1 to 4 .

I wish to thank the referee for his detailed comments on the exposition.

\section{The principle of construction of $f$ and $g$}

2.1. We explain the construction of $f$ in detail. The construction of $g$ is similar. The proofs of the theorems are based on this construction.

So $f$ is to be odd and piecewise linear, with increasing slopes for $x \geqq-1$, and with $f(x)=x$ for $-1 \leqq x \leqq 1$. Following [2, Section 7], we will call the points, where the slope of $f$ changes, critical points. Below, also some other points, where the slope is redefined even though it might not always change, will be called critical.

The positive critical points are denoted by $x_{n}, n \geqq 0, x_{0}=1$, and the slope between $x_{n-1}$ and $x_{n}$ is denoted by $s_{n}$. We set $s_{0}=1$, and we take $s_{1}=K, x_{1}=3$. Thus by $f(-x)=-f(x), f$ is now defined for $|x| \leqq 3$.

Each slope $s_{n}, n \geqq 1$, will be of the form $K^{p} L^{q}$ for integers $p \geqq 1, q \geqq 0$. We define $p_{n}^{\prime}, q_{n}^{\prime}$ so that $s_{n}=K^{p_{n}^{\prime}} L^{q_{n}^{\prime}}$. We set $p_{1}^{\prime}=1, q_{1}^{\prime}=0$.

Suppose that $x_{i}$ and $s_{i}$ have been defined for $1 \leqq i \leqq n$ and that $s_{i} \leqq s_{i+1}$ for all $i$. Thus $f$ is defined for $|x| \leqq x_{n}$. Suppose further that the construction has been made so that for every $N, 1 \leqq N \leqq n$, there are $i=i_{N}$ and $j=j_{N}$ such that $0 \leqq i$, $j<N$,

and

where

$$
x_{N}-x_{i}=x_{i}-\left(-x_{j}\right)
$$

$$
f\left(-x_{j}, x_{i}, x_{N}\right)=K \text {, }
$$

$$
f(x, y, z)=[f(z)-f(y)] /[f(y)-f(x)] .
$$

For $N=1$, we can take $i_{1}=j_{1}=0$, since

$$
f\left(-x_{0}, x_{0}, x_{1}\right)=f(-1,1,3)=K .
$$

We must define $s_{n+1} \geqq s_{n}$ and $x_{n+1}$, keeping in mind that $f$ is to be $K$-qs. Set $i=i_{n}, j=j_{n}$ so that $x_{n}-x_{i}=x_{i}+x_{j}$ and

$$
f\left(-x_{j}, x_{i}, x_{n}\right)=K \text {. }
$$


Thus we require that

$$
f\left(-x_{j+1}, x_{i}, x_{n}+\left(x_{j+1}-x_{j}\right)\right) \leqq K
$$

and

$$
f\left(-x_{j}, x_{i+1}, x_{n}+2\left(x_{i+1}-x_{i}\right)\right) \leqq K .
$$

Since the slopes are to be increasing, we are going to have $f\left(x_{n}+A\right) \geqq f\left(x_{n}\right)+A s_{n+1}$, $A \geqq 0$.

We can write (2.2) as

$$
\begin{gathered}
f\left(x_{n}+\left(x_{j+1}-x_{j}\right)\right)-f\left(x_{n}\right)+f\left(x_{n}\right)-f\left(x_{i}\right) \\
\leqq K\left(f\left(x_{i}\right)+f\left(x_{j+1}\right)\right)=K\left\{f\left(x_{i}\right)+f\left(x_{j}\right)-\left[f\left(x_{j}\right)-f\left(x_{j+1}\right)\right]\right\} .
\end{gathered}
$$

By (2.1), this reads

$$
\left(x_{j+1}-x_{j}\right) s_{n+1} \leqq K s_{j+1}\left(x_{j+1}-x_{j}\right),
$$

i.e., $s_{n+1} \leqq K s_{j+1}$. Similarly, (2.3) gives

$$
\begin{gathered}
2\left(x_{i+1}-x_{i}\right) s_{n+1}+K\left(f\left(x_{i}\right)+f\left(x_{j}\right)\right)-\left(f\left(x_{i+1}\right)-f\left(x_{i}\right)\right) \\
\leqq K\left\{f\left(x_{i+1}\right)-f\left(x_{i}\right)+f\left(x_{i}\right)+f\left(x_{j}\right)\right\}
\end{gathered}
$$

or $s_{n+1} \leqq(K+1) s_{i+1} / 2$. We take $s_{n+1}$ to be as large as to satisfy these two conditions, i.e.,

$$
s_{n+1}=\min \left(K s_{j+1},(K+1) s_{i+1} / 2\right) .
$$

If $K s_{j+1}<L s_{i+1}$, where $L=(K+1) / 2$, or if $K s_{j+1}=L s_{i+1}$ and $p_{j+1}^{\prime}+1 \geqq p_{i+1}^{\prime}$, we set $x_{n+1}=x_{n}+\left(x_{j+1}-x_{j}\right), i_{n+1}=i_{n}, j_{n+1}=j_{n}+1, p_{n+1}^{\prime}=p_{j+1}^{\prime}+1, q_{n+1}^{\prime}=q_{j+1}^{\prime}$, and we say that the slope $s_{n+1}$ was constructed from $s_{j+1}$. If $L s_{i+1}<K s_{j+1}$, or if $L s_{i+1}=$ $K s_{j+1}$ and $p_{j+1}^{\prime}+1<p_{i+1}^{\prime}$, we set $x_{n+1}=x_{n}+2\left(x_{i+1}-x_{i}\right), i_{n+1}=i_{n}+1, j_{n+1}=j_{n}$, $p_{n+1}^{\prime}=p_{i+1}^{\prime}, q_{n+1}^{\prime}=q_{i+1}^{\prime}+1$, and say that $s_{n+1}$ was constructed from $s_{i+1}$. In the former case, we have $f\left(-x_{j+1}, x_{i}, x_{n+1}\right)=K$, and in the latter case

$$
f\left(-x_{j}, x_{i+1}, x_{n+1}\right)=K,
$$

as required to continue the construction. Clearly $s_{n+1}=K^{p} L^{q}$ for $p=p_{n+1}^{\prime}, q=q_{n+1}^{\prime}$.

To prove that $s_{n+1} \geqq s_{n}$, we note that this is equivalent to

$$
K s_{j} \leqq \min \left(K s_{j+1}, L s_{i+1}\right),
$$

if $s_{n}=K s_{j}$, in which case $K s_{j} \leqq L s_{i+1}$ by the definition of $s_{n}$, and to

$$
L s_{i} \leqq \min \left(K s_{j+1}, L s_{i+1}\right)
$$

if $s_{n}=L s_{i}$, in which case $L s_{i} \leqq K s_{j+1}$. But $s_{i} \leqq s_{i+1}$ and $s_{j} \leqq s_{j+1}$ by assumption, so that (2.4) and (2.5) are true, and thus $s_{n+1} \geqq s_{n}$. Since $x_{n+1}-x_{n} \geqq 2$, this construction defines an increasing homeomorphism $f$ of $R$.

The fact that $f$ is the function defined in Section 1 by means of (1.3) and (1.4) will be proved in subsection 4.2 . 
2.2. The function $g$ is constructed by taking $g(x)=x$ for $-1 \leqq x \leqq 1, g(-x)=$ $-g(x)$, and by defining critical points $x_{n}$ and slopes $s_{n}$ as before, with $x_{0}=s_{0}=1$, $x_{1}=3, s_{1}=1 / K$. Suppose that $x_{i}$ and $s_{i}, 1 \leqq i \leqq n$, have been defined with $s_{i+1} \leqq s_{i}$ for all $i$, and suppose that $f\left(-x_{j}, x_{i}, x_{n}\right)=1 / K$ for some $i, j$ with $0 \leqq i, j<n$ and $x_{n}-x_{i}=x_{i}+x_{j}$. We note that $f\left(-x_{0}, x_{0}, x_{1}\right)=f(-1,1,3)=1 / K$. We also define $p_{n}^{\prime}, q_{n}^{\prime}$ so that $s_{n}=K^{-p_{n}^{\prime}} \Lambda_{n}^{q_{n}^{\prime}}$.

To define $s_{n+1}$, we note that the conditions corresponding to (2.2) and (2.3) are

$$
\begin{aligned}
& f\left(-x_{j+1}, x_{i}, x_{n}+\left(x_{j+1}-x_{j}\right)\right) \geqq 1 / K, \\
& f\left(-x_{j}, x_{i+1}, x_{n}+2\left(x_{i+1}-x_{i}\right)\right) \geqq 1 / K,
\end{aligned}
$$

which lead to $s_{n+1} \geqq s_{j+1} / K, s_{n+1} \geqq \Lambda s_{i+1}$, respectively, where $\Lambda=(1+1 / K) / 2$ as before. We set $s_{n+1}=\max \left(s_{j+1} / K, \Lambda s_{i+1}\right)$. If $s_{j+1} / K>\Lambda s_{i+1}$, or if $s_{j+1} / K=$ $\Lambda s_{i+1}$ and $p_{j+1}^{\prime}+1 \geqq p_{i+1}^{\prime}$, we set $x_{n+1}=x_{n}+\left(x_{j+1}-x_{j}\right)$ and define $p_{n+1}^{\prime}, q_{n+1}^{\prime}$ as before, and otherwise we set $x_{n+1}=x_{n}+2\left(x_{i+1}-x_{i}\right)$. Thus we have

$$
f\left(-x_{j+1}, x_{i}, x_{n+1}\right)=1 / K
$$

in the former case, and $f\left(-x_{j}, x_{i+1}, x_{n+1}\right)=1 / K$ in the latter case. It also follows that $s_{n+1} \leqq s_{n}$, so that the construction can proceed in the same way. This completes the construction of $g$.

2.3. For later reference we summarize the main points in the construction of $f$. If $i=i_{n}, j=j_{n}$, and $K s_{j+1}<L s_{i+1}$, or $K s_{j+1}=L s_{i+1}$ and $p_{j+1}^{\prime}+1 \geqq p_{i+1}^{\prime}$, we set

$$
\begin{gathered}
i_{n+1}=i_{n}, \quad j_{n+1}=j_{n}+1, \quad p_{n+1}^{\prime}=p_{j+1}^{\prime}+1, \quad q_{n+1}^{\prime}=q_{j+1}^{\prime}, \\
s_{n+1}=K s_{j+1}, \quad x_{n+1}=x_{n}+\left(x_{j+1}-x_{j}\right),
\end{gathered}
$$

while otherwise we set

$$
\begin{gathered}
i_{n+1}=i_{n}+1, \quad j_{n+1}=j_{n}, \quad p_{n+1}^{\prime}=p_{i+1}^{\prime}, \quad q_{n+1}^{\prime}=q_{i+1}^{\prime}+1, \\
s_{n+1}=L s_{i+1}, \quad x_{n+1}=x_{n}+2\left(x_{i+1}-x_{i}\right) .
\end{gathered}
$$

This implies that $i_{n} \rightarrow \infty$ and $j_{n} \rightarrow \infty$ as $n \rightarrow \infty$. Since every new slope is obtained by multiplying a previous slope by $K$ or $L$, it follows that every slope is of the form $K^{p} L^{q}$ for integers $p \geqq 1, q \geqq 0$. We have $p \geqq 1$ since $s_{1}=K$.

By inspection of the construction of $f$, we observe that every interval $\left[x_{m-1}, x_{m}\right]$ is used twice in the construction of $f$ on a new interval $\left[x_{n-1}, x_{n}\right]$. This happens when $i_{n}=m$ and when $j_{n}=m$. We also see that every number $K^{p} L^{q}$ as above occurs as a slope of $f$ on some interval.

Similarly, every slope of $g$ is of the form $K^{-P} \Lambda^{Q}$ for integers $P \geqq 1, Q \geqq 0$, and every such number $K^{-P} \Lambda^{Q}$ occurs as a slope of $g$ on some interval. 


\section{Proof that $f$ and $g$ are $K$-qs}

3.1. We have to show that $f, g \in N_{0}(K)$. Clearly $f(-1)=g(-1)=-1$ and $f(1)=g(1)=1$. We show that $f$ is $K$-qs. For $g$, the proof is similar and we omit it.

By [2, Lemma 4] it suffices to show that $1 / K \leqq f(x, y, z) \leqq K$ when $z-y=$ $y-x>0$ and at least two of the points $x, y, z$ are critical. By symmetry, we may assume that $y>0$. There are six cases to consider:

$1^{0}$

$2^{0}$

$3^{0}$

$4^{0}$

$5^{0}$

$6^{0}$

$$
\begin{gathered}
x=x_{j}, \quad y=x_{i}, \quad z=2 x_{i}-x_{j}, \quad j+1 \leqq i, \\
-1 \leqq x=2 x_{i}-x_{j}, \quad y=x_{i}, \quad z=x_{j}, \quad i+1 \leqq j, \\
x=x_{j}, \quad y=\frac{1}{2}\left(x_{i}+x_{j}\right), \quad z=x_{i}, \quad j+1 \leqq i, \\
x=-x_{j}, \quad y=x_{i}, \quad z=2 x_{i}+x_{j}, \\
x=2 x_{i}-x_{j}<-1, \quad y=x_{i}, \quad z=x_{j}, \quad i+1 \leqq j, \\
x=-x_{j}, y=\frac{1}{2}\left(x_{i}-x_{j}\right), \quad z=x_{i}, \quad j<i .
\end{gathered}
$$

Some of these cases reduce to others, and some of them are so similar that we do not treat all of them in detail.

3.2. Since the slopes of $f$ are increasing for $x \geqq-1$, it follows that $f(x, y, z) \geqq 1$ in all the cases $1^{0}$ to $6^{0}$. If $x \geqq 0$, this is obvious. If $x<0$, then, since $y>0$, we have $f(z)-f(y) \geqq f(z-y)$ and $f(y)-f(x)=f(y)+f(|x|) \leqq f(y+|x|)=f(y-x)$. Since $z-$ $y=y-x$, this implies that $f(x, y, z) \geqq 1$. It remains to find an upper bound for $f(x, y, z)$.

3.3. Consider the case $1^{0}$, and let $i$ be fixed. Since the slopes of $f$ are increasing for $x \geqq-1$, the functions $\left[f\left(x_{i}+t\right)-f\left(x_{i}\right)\right] t^{-1}$ and $t\left[f\left(x_{i}\right)-f\left(x_{i}-t\right)\right]^{-1}$ are increasing functions of $t$ for $0<t \leqq x_{i}+1$. Thus to get an upper bound for $f\left(x_{j}, x_{i}, 2 x_{i}-x_{j}\right)$ for this fixed $i$ it suffices to replace $x_{j}$ by $-1=-x_{0}$. Hence $1^{0}$ reduces to $4^{0}$.

Consider $2^{0}$ for a fixed $i$. The previous argument shows that to get an upper bound for any $j$ considered, it suffices to replace $x$ by -1 and $z$ by $2 x_{i}+1$. Thus $2^{0}$ reduces to $4^{0}$.

3.4. Consider $3^{0}$ for fixed $i$ and $j$. We want to show that if $f(x, y, z) \leqq K$ for $x, y, z$ as in $4^{0}$ and thus as in $1^{0}$, then the same is true in $3^{0}$.

If $y=x_{m}$ for some $m$, then $3^{0}$ reduces to $1^{0}$ and thus to $4^{0}$. If not, suppose that $x_{m}<y<x_{m+1}$, and write $s_{m+1}=S, y-x_{m}=\delta_{1}$.

First we show that $s_{i} \leqq L S$. There are $l \geqq 0$ and $q>m$ such that $x_{q}-x_{m}=$ $x_{m}+x_{l}$ and $f\left(-x_{l}, x_{m}, x_{q}\right)=K$. We can choose $q$ and $l$ so that $x_{q+1}-x_{q}=$ 
$2\left(x_{m+1}-x_{m}\right)$ and $s_{q+1}=L s_{m+1}$, by taking $q$ and $l$ larger than originally, if necessary. Further, we have

and hence

$$
x_{i}-x_{m}=x_{m}-x_{j}+2\left(y-x_{m}\right) \leqq x_{m}-x_{j}+2\left(x_{m+1}-x_{m}\right)
$$

$$
z=x_{i} \leqq 2 x_{m}+x_{q+1}-x_{q}=x_{q}-x_{l}+x_{q+1}-x_{q} \leqq x_{q+1},
$$

so that $s_{i} \leqq s_{q+1}=L s_{m+1}=L S$.

Returning to $f(x, y, z)$, we note that if $x_{j}<x_{m}$ and if $f(x, y, z) \leqq K$ in $1^{0}$, then

$$
f\left(x_{j}, x_{m}, x_{i}-2 \delta_{1}\right) \leqq K,
$$

which reads for the present $x, y, z$,

Hence

$$
f\left(z-2 \delta_{1}\right)-f(z)+f(z)-f(y)+\delta_{1} S \leqq K\left\{f(y)-f(x)-\delta_{1} S\right\} .
$$

$$
f(z)-f(y) \leqq K\{f(y)-f(x)\}+\left\{f(z)-f\left(z-2 \delta_{1}\right)\right\}-2 \delta_{1} L S .
$$

Since $z=x_{i} \leqq x_{q+1}$, the slopes of $f$ up to $z$ do not exceed $s_{q+1} \leqq L S$, which implies that

$$
f(z)-f\left(z-2 \delta_{1}\right) \leqq 2 \delta_{1} L S .
$$

This together with (3.1) gives $f(x, y, z) \leqq K$.

If $x_{j}=x_{m}$, it suffices to show that the ratio of the largest and smallest slopes of $f$ on $\left[x_{m}, z\right]$ does not exceed $K$. This ratio is at most $s_{q+1} / s_{m+1}=L<K$. Thus $f(x, y, z) \leqq K$ also in this case.

3.5. Consider $4^{0}$ for a fixed $i$. First we take $j=l$ so that for some $n, x_{n}-x_{i}=$ $x_{i}+x_{l}$ and $f\left(x_{0}, y, z_{0}\right)=K$, where $x_{0}=-x_{l}, y=x_{i}, z_{0}=2 y-x_{0}=x_{n}$. Suppose then that $x=-x_{j}$ for some $j, 0 \leqq j<l$ and that $z=2 y-x$. From the construction of $f$ it follows that for every interval $\left(-x_{m},-x_{m-1}\right), 1 \leqq m \leqq l$, there is an interval $I_{m}$ of length $x_{m}-x_{m-1}$ contained in $\left[1, z_{0}\right]$ such that $I_{m} \cap I_{k}=\emptyset$ for $m \neq k$, the left endpoint of $I_{m}$ is smaller than that of $I_{k}$ if $m<k$, and the slope of $f$ on $I_{m}$ equals $K s_{m}$. Between $I_{m}$ and $I_{m+1}$, the slopes of $f$ lie between $K s_{m}$ and $K s_{m+1}$. It follows that

$$
f\left(z_{0}\right)-f(z) \geqq K\left\{f(x)-f\left(x_{0}\right)\right\},
$$

so that since $f\left(z_{0}\right)-f(y)=K\left\{f(y)-f\left(x_{0}\right)\right\}$, we have

$$
f(z)-f(y) \leqq K\{f(y)-f(x)\} .
$$

Similarly for $m \geqq l+1$, there is an interval $I_{m}$ with the above properties contained in $\left[z_{0}, \infty\right)$. Therefore $f(z)-f\left(z_{0}\right) \leqq K\left\{f\left(x_{0}\right)-f(x)\right\}$, so that $f(x, y, z) \leqq K$.

This gives the desired upper bound in the case $4^{0}$, and the case $5^{0}$ is similar.

3.6. Consider $6^{0}$ for a fixed $j$. Note that $x_{j}-x_{i} \geqq 2$ for $j>i$ so that $y \geqq 1$. First we take $i$ so that with $z_{0}=x_{i}$ and $z_{0}-y_{0}=y_{0}-x, x=-x_{j}$, we have $y_{0}=x_{l}$ for some $l$ and $f\left(x, y_{0}, z_{0}\right)=K$. For every interval $\left(x_{m}, x_{m+1}\right), m \geqq l$, there is an interval $I_{m} \subset\left[z_{0}, \infty\right)$ of length $2\left(x_{m+1}-x_{m}\right)$ such that $I_{m} \cap I_{k}=\emptyset$ for $m \neq k$, the 
left endpoint of $I_{m}$ is smaller than that of $I_{k}$ if $m<k$, and the slope of $f$ on $I_{m}$ equals $L s_{m+1}$. For $0 \leqq m \leqq l$, there are intervals $I_{m} \subset\left[1, z_{0}\right]$ with the same properties. It follows that if $z>z_{0}$, then

and if $z<z_{0}$, then

$$
f(z)-f\left(z_{0}\right) \leqq 2 L\left\{f(y)-f\left(y_{0}\right)\right\},
$$

$$
f\left(z_{0}\right)-f(z) \geqq 2 L\left\{f\left(y_{0}\right)-f(y)\right\},
$$

so that in any case $f(x, y, z) \leqq K$. Thus $f$ is $K$-qs.

\section{Proof of Theorem 1}

4.1. We have already proved that $f, g \in N_{0}(K)$. Next we show that

$$
f\left(x_{n}\right)=M_{0}\left(x_{n}, K\right)
$$

for the critical points $x_{n}$ of $f$. The proof is by induction. For $n=0,1,(4.1)$ is true. Suppose that (4.1) holds for $n \leqq k$. Then with $i=i_{k+1}, j=j_{k+1}$ we have $0 \leqq i, j \leqq k$, $x_{k+1}-x_{i}=x_{i}+x_{j}$ and

$$
f\left(x_{k+1}\right)=f\left(x_{i}\right)+K\left\{f\left(x_{i}\right)+f\left(x_{j}\right)\right\} .
$$

Suppose that $h \in N_{0}(K)$. Then by (1.1),

$$
\begin{aligned}
h\left(x_{k+1}\right) & \leqq h\left(x_{i}\right)+K\left\{h\left(x_{i}\right)-h\left(-x_{j}\right)\right\} \\
& \leqq M_{0}\left(x_{i}, K\right)+K\left\{M_{0}\left(x_{i}, K\right)+M_{0}\left(x_{j}, K\right)\right\},
\end{aligned}
$$

since also $-h(-x) \in N_{0}(K)$. By the induction assumption, the right hand sides of (4.2) and (4.3) are equal, so that $h\left(x_{k+1}\right) \leqq f\left(x_{k+1}\right)$ for all $h \in N_{0}(K)$ and so $M_{0}\left(x_{k+1}, K\right) \leqq f\left(x_{k+1}\right)$. The reverse inequality follows from the definition of $M_{0}(x, K)$ since $f \in N_{0}(K)$, so that (4.1) holds for $n=k+1$. This completes the induction. Similarly it is proved that $g\left(x_{n}\right)=m_{0}\left(x_{n}, K\right)$ for the critical points $x_{n}$ of $g$.

4.2. Next we show that the function $f$ constructed in Section 2 is the same as that defined in Section 1 by means of (1.3) and (1.4). To do this, we prove that $f$ as defined in Section 2 has certain properties.

Let the pairs $\left(p_{k}, q_{k}\right)$ be as in (1.2), and let $\left(p_{k}^{\prime}, q_{k}^{\prime}\right)$ be as defined in subsection 2.1. Recall that if $K^{p_{k}} L^{q_{k}}$ assumes the same value for several pairs $\left(p_{k}, q_{k}\right)$, say for $M \leqq k \leqq N$, then these pairs occur in the descending order of $p_{k}$, i.e., $p_{M}>$ $p_{M+1}>\ldots>p_{N}$. We make the following induction assumption, which is clearly true for $n=1$. In the list of pairs $\left(p_{k}^{\prime}, q_{k}^{\prime}\right), k \geqq 1$, we first have $\left(p_{1}, q_{1}\right)$, then $\left(p_{2}, q_{2}\right)$ repeated $N_{2}$ times, and so on, up to $\left(p_{n}, q_{n}\right)$ repeated $N_{n}$ times, where $N_{k}=\left(\begin{array}{l}R \\ S\end{array}\right)$, $R=p_{k}+q_{k}-1, S=q_{k}$. Further, if the slope of $f$ is $K^{p} L^{q}$ between successive critical 
points $x$ and $y$, where $x<y \leqq x_{n}$, then

$$
y-x=2^{q+1} .
$$

We assume that this is true up to a certain $n \geqq 1$, and prove that it remains valid if $n$ is replaced by $n+1$.

We recall that every number $K^{p} L^{q}, p \geqq 1, q \geqq 0$, occurs as a slope of $f$, as observed in subsection 2.3. We set $p_{n+1}=P, q_{n+1}=Q$. The new slope $K^{P} L^{Q}$ can be constructed from earlier slopes $K^{P-1} L^{Q}$ if $P \geqq 2$, and from $K^{P} L^{Q-1}$ if $Q \geqq 1$. In any case $P \geqq 2$ or $Q \geqq 1$, for otherwise $P=1, Q=0$, hence $n+1=1$, which is against our assumption. We assume that $P \geqq 2$ and $Q \geqq 1$, for if this is not the case, then the conclusion is obtained by omitting parts of the following argument. If the old slope used is $K^{P-1} L^{Q}$, then the distance between the new critical points is equal to the earlier one, i.e., $2^{Q+1}$. If the old slope is $K^{P} L^{Q-1}$, then the new distance is twice the old one, which is $2^{(Q-1)+1}$, so that the new distance is again $2^{Q+1}$. The number of intervals $\left[x_{m-1}, x_{m}\right]$ with the slope $K^{P-1} L^{Q}$ and with $p_{m}^{\prime}=P-1, q_{m}^{\prime}=Q$ is $\left(\begin{array}{c}P+Q-2 \\ Q\end{array}\right)$, and the number of those with the slope $K^{P} L^{Q-1}$ and $p_{m}^{\prime}=P, q_{m}^{\prime}=$ $Q-1$ is $\left(\begin{array}{r}P+Q-2 \\ Q-1\end{array}\right)$, so that the number of new intervals $\left[x_{m-1}, x_{m}\right]$ with the slope $K^{P} L^{Q}$ and with $p_{m}^{\prime}=P, q_{m}^{\prime}=Q$ is

$$
\left(\begin{array}{c}
P+Q-2 \\
Q
\end{array}\right)+\left(\begin{array}{c}
P+Q-2 \\
Q-1
\end{array}\right)=\left(\begin{array}{c}
P+Q-1 \\
Q
\end{array}\right)
$$

If $K^{p} \neq L^{q}$ for all $p, q$, there is nothing else to prove. Suppose that $K^{r}=L^{s}$ for some relatively prime integers $r, s \geqq 1$. Then $K^{P} L^{Q}=K^{P-r} L^{Q+s}=\ldots=K^{P-u r} L^{Q+u s}$ where $P-u r \geqq 1>P-(u+1) r$, and $K^{P} L^{Q}=K^{P+r} L^{Q-s}=\ldots=K^{P+v r} L^{Q-v s}$, where $Q-v s \geqq 0>Q-(v+1) s$. By the induction assumption, the pairs $(P+v r, Q-v s), \ldots$, $(P+r, Q-s)$, each repeated a certain number of times, precede $(P, Q)$ among the $\left(p_{k}^{\prime}, q_{k}^{\prime}\right)$. Now among the pairs $\left(p_{k}^{\prime}, q_{k}^{\prime}\right)$ for which $K^{p_{k}^{\prime}} L^{q_{k}^{\prime}}=K^{P-1} L^{Q}$, we have the pairs $(P-1, Q),(P-1-r, Q+s), \ldots$ in this order, by the induction assumption. The pairs $(P-1+r, Q-s)$ etc. have already been used to obtain $(P+r, Q-s)$ etc. Similarly, among the pairs $\left(p_{k}^{\prime}, q_{k}^{\prime}\right)$ with $K^{p_{k}^{\prime}} L^{q_{k}^{\prime}}=K^{P} L^{Q-1}$ we have $(P, Q-1)$, $(P-r, Q-1+s)$ etc. left in this order. Following the procedure of constructing $f$ summarized in 2.3 , when constructing the slope $K^{P} L^{Q}$ we first have a choice between using $(P-1, Q)$ and $(P, Q-1)$, and we first use up the pairs $(P-1, Q)$. Then we have a choice between $(P-1-r, Q+s)$ and $(P, Q-1)$, and we use the pairs $(P, Q-1)$. Continuing in this way we see that the pairs $(P, Q),(P-r, Q+s)$ etc. in this order, each repeated a certain number of times, are among the $\left(p_{k}^{\prime}, q_{k}^{\prime}\right)$. This completes the induction.

From what has been proved above it is obvious that the function $f$ constructed in Section 2 is indeed the same as that defined in Section 1 by means of (1.3) and (1.4), and that (1.8) holds for the points $x$ mentioned in Theorem 1. For $g$, the argument is similar. Theorem 1 is proved. 


\section{Proof of Theorems 2 and 3}

Proof of Theorem 2. For $x \geqq-1$, the function $f$ is clearly a convex minorant of $M_{0}(x, K)$, since $f \in N_{0}(K)$. Suppose that $h$ is a convex minorant of $f$ for $x \geqq-1$, and that $x$ and $y$ are two successive critical points of $f$. Then

$$
h(x) \leqq M_{0}(x, K)=f(x), \quad h(y) \leqq M_{0}(y, K)=f(y) .
$$

Since $f$ is linear between $x$ and $y$ and $h$ is convex, we have $h(t) \leqq f(t)$ for $x \leqq t \leqq y$. Thus $f$ is the largest convex minorant of $M_{0}(x, K)$.

Similarly it is proved that $g$ is the smallest concave majorant of $m_{0}(x, K)$. Theorem 2 is proved.

Proof of Theorem 3. We consider $f$ only, since the proof for $g$ is similar. The proof is the same as in [2, Theorem 13]. Suppose that $f$ has left and right hand derivatives $s_{1}$ and $s_{2}$ at $X_{n}, s_{1}<s_{2}$. If $M_{0}(x, K)$ is differentiable at $x=X_{n}$ with left and right hand derivatives $D_{-}$and $D_{+}$, then $f\left(X_{n}\right)=M_{0}\left(X_{n}, K\right)$ and $f(x) \leqq$ $M_{0}(x, K)$ for all $x$ imply that $D_{+} \geqq s_{2}$ and $D_{-} \leqq s_{1}$. Thus $D_{-}<D_{+}$, which is a contradiction. Theorem 3 is proved.

\section{Proof of Theorem 4}

Consider first $f$. It follows from (1.10) that $K^{q}=L^{p}$. Note that $1<p / q<2$, so $q \geqq 2$. We consider the solutions $z$ of

$$
f(z)=M_{0}(z, K),
$$

and prove the following lemma.

Lemma 1. Let $\left[v_{1}, v_{2}\right],\left[v_{3}, v_{4}\right],\left[v_{5}, v_{6}\right]$ be the intervals on the positive axis where the slope of $f$ equals $K^{P-1} L^{Q}, K^{P} L^{Q-1}, K^{P} L^{Q}$, respectively. Suppose that (6.1) holds for $z=z_{k}, 1 \leqq k \leqq N$, and for $z=u_{k}, 1 \leqq k \leqq M$, where $v_{1}=z_{1}<z_{2}<\ldots<$ $z_{N}=v_{2}$ and $v_{3}=u_{1}<u_{2}<\ldots<u_{M}=v_{4}$. Then if $1 \leqq n \leqq N, 1 \leqq m \leqq M$, the point

$$
z=v_{5}+\left(z_{n}-z_{1}\right)+2\left(u_{m}-u_{1}\right)
$$

is a solution of (6.1).

We have $f\left(v_{5}\right)-f\left(v_{3}\right)=K\left\{f\left(v_{3}\right)+f\left(v_{1}\right)\right\}$ by the definition of $f$. Using this together with the slopes of $f$ on the three intervals, we get

$$
f(z)-f\left(v_{7}\right)=K\left\{f\left(v_{7}\right)+f\left(v_{8}\right)\right\}
$$

where $z$ is given by (6.2), $v_{7}=v_{3}+\left(u_{m}-u_{1}\right)=u_{m}$ and $v_{8}=v_{1}+\left(z_{n}-z_{1}\right)=z_{n}$. Since $z=u_{m}$ and $z=z_{n}$ satisfy (6.1), the argument used in 4.1 shows that (6.1) holds for $z$ given by (6.2). This proves Lemma 1 . 
We shall now establish the upper bound (1.11) for the distance between two successive solutions of (6.1).

We set $V=2^{p}+p-1$. We use $Z_{1}, Z_{2}$ and $d=Z_{2}-Z_{1}>0$ as a generic notation for two successive known solutions of (6.1) and their distance.

The value of each slope can be written uniquely as $K^{r} L^{N}, N \geqq 0,1 \leqq r \leqq q$. We want to prove that $d \leqq 2^{V+1}$. If $s$, the slope between $Z_{1}$ and $Z_{2}$, satisfies $s=K^{r} L^{N}$, $N \leqq V$, then $d \leqq 2^{V+1}$. Namely, among all pairs $(t, w)$ such that $s=K^{t} L^{w}, t=r$ is minimal and hence $w=N$ is maximal. On the intervals where the slope of $f$ is $s$, any critical points of $f$ are solutions of (6.1) by 4.1, and by (4.4) the distance between two successive critical points on such intervals is at most $2^{w+1} \leqq 2^{N+1} \leqq$ $2^{V+1}$. The proof that $d \leqq 2^{V+1}$ in general is by induction over the pairs $(r, N)$, with respect to the order of pairs defined by

$$
\left(r_{1}, N_{1}\right)<\left(r_{2}, N_{2}\right) \text { if } N_{1}<N_{2} \text { or if } N_{1}=N_{2} \text { and } r_{1}<r_{2} .
$$

Only the pairs $(r, N)$ with $N \leqq V$ are considered. Note that $1 \leqq r \leqq q$ for all $(r, N)$.

For $(r, N)=(1, V)$ we have $d \leqq 2^{V+1}$, as mentioned above. Suppose that this is true for all pairs before a certain pair $(r, N)>(1, V)$, and let us prove it for $(r, N)$. We assume that $\left[v_{1}, v_{2}\right],\left[v_{3}, v_{4}\right],\left[v_{5}, v_{6}\right]$ are the intervals on the positive axis where the slope of $f$ is $K^{r-1} L^{N}, K^{r} L^{N-1}, K^{r} L^{N}$, respectively. If $r=1$, we replace $K^{r-1} L^{N}$ by $K^{q} L^{N-p}$. Note that then $N \geqq V+1$, hence $N-p \geqq 2^{p}+p-p>0$.

By assumption, we have $d \leqq 2^{V+1}$ on $\left[v_{1}, v_{2}\right]$ and $\left[v_{3}, v_{4}\right]$. Hence there are points $z_{1}=v_{1}<z_{2}<\ldots<z_{N}=v_{2}$ and $u_{1}=v_{3}<u_{2}<\ldots<u_{M}=v_{4}$, all of which satisfy (6.1). We assume that these include all critical points of $f$ on these intervals. Further, we have $z_{i+1}-z_{i} \leqq 2^{V+1}, u_{j+1}-u_{j} \leqq 2^{V+1}$ for $1 \leqq i<N, 1 \leqq j<M$. By Lemma 1 , the points

$$
w_{m n}=v_{5}+2\left(u_{m}-u_{1}\right)+\left(z_{n}-z_{1}\right), \quad 1 \leqq m \leqq M, \quad 1 \leqq n \leqq N,
$$

are solutions of (6.1). Let $m$ be fixed and let $n$ vary from 1 to $N$. We see that $d \leqq 2^{V+1}$ on $\left[w_{M 1}, v_{6}\right]$ in any case, and on $\left[v_{m 1}, w_{m+1,1}\right]$ for $1 \leqq m<M$ provided that $z_{N}-z_{1}$ $=v_{2}-v_{1} \geqq 2\left(u_{m+1}-u_{m}\right)$, hence on $\left[v_{5}, v_{6}\right]$ since each $w_{m 1}$ satisfies (6.1).

It remains to show that $2\left(u_{m+1}-u_{m}\right) \leqq v_{2}-v_{1}$. Since $\left[u_{m}, u_{m+1}\right] \subset\left[v_{3}, v_{4}\right]$, where the slope of $f$ is $K^{r} L^{N-1}$, we have anyway $u_{m+j}-u_{m} \leqq 2^{(N-1)+1}=2^{N}$ (cf. (4.4)). Also $\left[v_{1}, v_{2}\right]$ contains at least the intervals $\left[x_{n-1}, x_{n}\right]$ corresponding to the pairs $\left(p_{n}^{\prime}, q_{n}^{\prime}\right)=(r-1, N)$ if $r-1 \geqq 1$, or to $\left(p_{n}^{\prime}, q_{n}^{\prime}\right)=(q, N-p)$. Considering the number and length of such intervals, as obtained in 4.2 , we see that

$$
v_{2}-v_{1} \geqq\left(\begin{array}{c}
r-1+N-1 \\
N
\end{array}\right) 2^{N+1} \geqq 2^{N+1} \geqq 2\left(u_{m+1}-u_{m}\right)
$$

if $r \geqq 2$, and otherwise

$$
v_{2}-v_{1} \geqq\left(\begin{array}{c}
q+N-p-1 \\
N-p
\end{array}\right) 2^{N-p+1} .
$$


We have $q-1 \geqq 1$ and so

$$
\begin{gathered}
\left(\begin{array}{c}
(N-p)+(q-1) \\
N-p
\end{array}\right)=\frac{(N-p+q-1) \ldots(q+1) q}{(N-p) !} \\
\geqq \frac{(N-p+1)(N-p) \ldots 2 \cdot 1}{(N-p) !}=N-p+1 \geqq V-p+1=2^{p} .
\end{gathered}
$$

Hence $v_{2}-v_{1} \geqq 2^{N+1} \geqq 2\left(u_{m+1}-u_{m}\right)$. This proves Theorem 4 for $f$.

For $g$ the argument is almost similar. Since

$$
\frac{\log (1 / K)}{\log \Lambda}=\frac{p}{p-q}>2,
$$

the role of $q$ is taken by $p-q$ and the inequality corresponding to (6.3) is

$$
v_{2}-v_{1} \geqq\left(\begin{array}{c}
N-p+(p-q-1) \\
N-p
\end{array}\right) 2^{N-p+1} \geqq 2^{N+1}
$$

for $N \geqq V=2^{2 p}+2 p-1$ if $p-q-1 \geqq 1$. But if $p-q=1=r$, we note that $\left[v_{1}, v_{2}\right]$ contains at least the intervals $\left[x_{n-1}, x_{n}\right]$ corresponding to the pairs $\left(p_{k}^{\prime}, q_{k}^{\prime}\right)=$ $(2, N-2 p)$ with the slope $K^{-2} \Lambda^{N-2 p}$, where $N-2 p \geqq V+1-2 p>0$, so that

$$
v_{2}-v_{1} \geqq\left(\begin{array}{c}
N-2 p+2-1 \\
N-2 p
\end{array}\right) 2^{N-2 p+1} \geqq(V+1-2 p) 2^{N-2 p+1}=2^{N+1},
$$

as required. Theorem 4 is proved.

\section{References}

[1] Beurling, A., and L. AhLfors: The boundary correspondence under quasiconformal mappings. - Acta Math. 96, 1956, 125-142.

[2] Hayman, W. K., and A. Hinkkanen: Distortion estimates for quasisymmetric functions. To appear in Ann. Univ. Mariae Curie-Sklodowska.

[3] Hayman, W. K.: The asymptotic behaviour of K.q.s functions. - To appear in Volume in honour of L. Iliev, Bulgarian Academy of Sciences.

Imperial College

Department of Mathematics

London SW7 2BZ

United Kingdom
Current address:

University of Michigan

Department of Mathematics

Ann Arbor, Michigan 48109

USA 